\title{
Traffic jam driving with NMV avoidance
}

\author{
Vicente Milanés Luciano Alonso Jorge Villagrá Jorge Godoy \\ Teresa de Pedro Juan P. Oria
}

\begin{abstract}
A B S T R A C T
In recent years, the development of advanced driver assistance systems (ADAS) - mainly based on lidar and cameras - has considerably improved the safety of driving in urban environments. These systems provide warning signals for the driver in the case that any unexpected traffic circumstance is detected. The next step is to develop systems capable not only of warning the driver but also of taking over control of the car to avoid a potential collision. In the present communication, a system capable of autonomously avoiding collisions in traffic jam situations is presented. First, a perception system was developed for urban situations - in which not only vehicles have to be considered, but also pedestrians and other non-motor-vehicles (NMV). It comprises a differential global positioning system (DGPS) and wireless communication for vehicle detection, and an ultrasound sensor for NMV detection. Then, the vehicle's actuators brake and throttle pedals - were modified to permit autonomous control. Finally, a fuzzy logic controller was implemented capable of analyzing the information provided by the perception system and of sending control commands to the vehicle's actuators so as to avoid accidents. The feasibility of the integrated system was tested by mounting it in a commercial vehicle, with the results being encouraging.
\end{abstract}

\section{Introduction}

Safety plays a key role in the automotive sector [1]. For this reason, a considerable number of driver assistance systems have been installed in commercial cars in this last decade. The aim of these systems is to control the car's actuators through sensor systems to prevent or mitigate collisions. For example, radar [2], vision-based [3], and differential global positioning system (DGPS) [4] technologies have been used to detect any leading vehicle so as to perform adaptive cruise control (ACC) - a now well-proven technique consisting of speed control to maintain a pre-defined time gap with a leading vehicle. Likewise; these technologies have been used for pre-crash systems development. These systems are able to detect imminent collisions, warning the driver and preparing both vehicle and passengers for crash [5]. These commercial systems are extensively used in motorway driving, and are designed for speeds greater than $30 \mathrm{~km} / \mathrm{h}$.

The next step is to obtain systems capable of working at lower speeds. Indeed, $90 \%$ of urban crashes occur at speeds less than $25 \mathrm{~km} / \mathrm{h}$ due to the driver's attention being distracted [6]. At these low speeds, most road accident victims are not the motor vehicle's occupants, but pedestrians, motorcyclists, cyclists, and non-motor-vehicle (NMV) occupants [7]. The main 
problem is the driver's difficulty in reacting sufficiently quickly to unexpected circumstances. Two of the hottest topics in the transportation field correspond to driving in urban environments-following a leading vehicle at low speeds and reacting quickly to NMVs. The solution will need to use sensory information to autonomously manage the actuators-i.e., the throttle and brake pedals. One of the most challenging design issues in the short-to-medium term is therefore to develop such a sensor system for urban traffic conditions. In this sense, Volvo has developed the City Safety system for its vehicles. This system helps driver to entirely avoid a collision - when vehicle speed is under $15 \mathrm{~km} / \mathrm{h}$ - or reduce as much as possible the speed prior crashing [8]. Moreover, Volvo has recently implemented a novel solution called Collision Warning with Auto Brake (CWAB) which helps drivers to avoid collisions at higher speeds - up to $30 \mathrm{~km} / \mathrm{h}$. The system merges the information of a long-range radar with a forward-sensing wide-angle camera to monitor the area in front of vehicle [9].

Four principal types of sensor technology have been used in approaches to solving the NMV detection problem: laser radar (lidar), microwave radar, infra-red thermal imaging, and machine vision. While under optimal conditions lidars have high accuracy, wide angle coverage, and precise target location, their performance is lowered in inclement weather and/or when dirt collects on the lenses [10]. Most radars use some form of beam scanning to resolve whether targets are in the same roadway lane, an adjacent lane, or an oncoming lane. While microwave radars are able to operate accurately in inclement weather, they have a narrow field of vision and are prohibitively expensive for mass-produced cars [11].

The images from a video camera are usually produced at a $25 \mathrm{~Hz}$ frame rate, and these frames can be stored and processed by a computer as a matrix of pixels. Image processing techniques are used to provide information about the presence of obstacles up to $50 \mathrm{~m}$ away [12]. However, as humans vary significantly in size and shape, there are difficulties in obtaining robust results with image analysis techniques, especially in crowded areas. Moreover, extreme lighting conditions (day/night) can dramatically reduce the effectiveness of the detection algorithms. While passive (thermal) infra-red imaging already provides night vision in commercial ADAS systems [13], the information it provides is neither trustworthy nor persistent enough to be used in automated cars.

Obstacle detection at short distances is usually approached using microwave radar, radio-frequency capacitance, infrared multi-beams, or ultrasound. Capacitance sensors measure the change in dielectric constant that occurs when an object whose dielectric properties are different from air approaches the sensor. They have yet to be implemented in massproduced vehicles because they are highly sensitive to radio-frequency interference.

Ultrasound technology is widely used in production for parking man?uvres because it offers wide-area, near-distance beam coverage, and is low-cost [14]. Ultrasound sensors can be used in air at a range of $15 \mathrm{~m}$ with simple and cheap electronics. Their main advantage over other types of sensor is that they can detect any NMV in the roadway, which makes them well suited to preventing, or at least minimizing, damage. Their main disadvantage is that they are influenced by turbulence caused by the wind which disperses the ultrasound waves or produces false echoes.

The present communication is part of a collaboration between the AUTOPIA program at the Centre for Automation and Robotics (CAR, CSIC-UPM) and Santander University. The goal is to develop an approach to ACC plus NMV detection in urban environments. To this end, a prototype vehicle equipped with a DGPS combined with an Inertial Measurement Unit (IMU) to obtain the vehicle's positioning with high accuracy has been instrumented with automatic driving capabilities. Using wireless communications, the vehicle can receive data on the position of the other vehicles in its vicinity to perform ACC man?uvres. An ultrasound sensor detects obstacles on the road. With these two systems, measurements can be made of the distance to any object located in front of the vehicle. If the distances determined by the GPS and the ultrasound sensor are equal, then a leading vehicle has been detected. If the distances are different, it is assumed that an NMV has been detected between the two motor vehicles. In a later phase of the research program - the control stage - a fuzzy logic controller is designed to act autonomously on the throttle and brake pedals.

The rest of the paper is structured as follows. Section 2 describes the system developed to control the vehicle, and Sections 3 and 4 present the sensor systems used to acquire environmental information on any leading vehicle and NMVs, respectively. Section 5 presents the fuzzy-based control algorithm, and Section 6 the brake and throttle automation system. Experimental results of trials on a private driving circuit are described in Section 7, and some concluding remarks are given in Section 8.

\section{The on-board system}

The control system is divided into three stages (see Fig. 1). The first stage is in charge of acquiring all the sensorial inputs from the environment. The vehicle is equipped with a differential global positioning system (DGPS) located close to the rear-end of the car, and an inertial measurement unit (IMU) located as close as possible to the centre of gravity. They are in charge of positioning the vehicle in the road [15]. The commercial Citroën C3's controller area network (CAN) bus is available to acquire information on the vehicle. For this application, only information coming from the speedometer is used. An ultrasound sensor is located on the front bonnet of the car. A Personal Computer Memory Card International Association (PCMCIA) Proxim Wireless ComboCard is used to obtain information about other vehicles in the vicinity. Sections 3 and 4 will describe the sensors used to carry out autonomous ACC with NMV detection, respectively.

The second stage is in charge of selecting and executing the best control strategy in each driving situation. To this end, different fuzzy controllers [4,16-18] have been developed to take into account various traffic situations. The fuzzy controller implemented in the proposed driving aid system will be described in Section 5. 


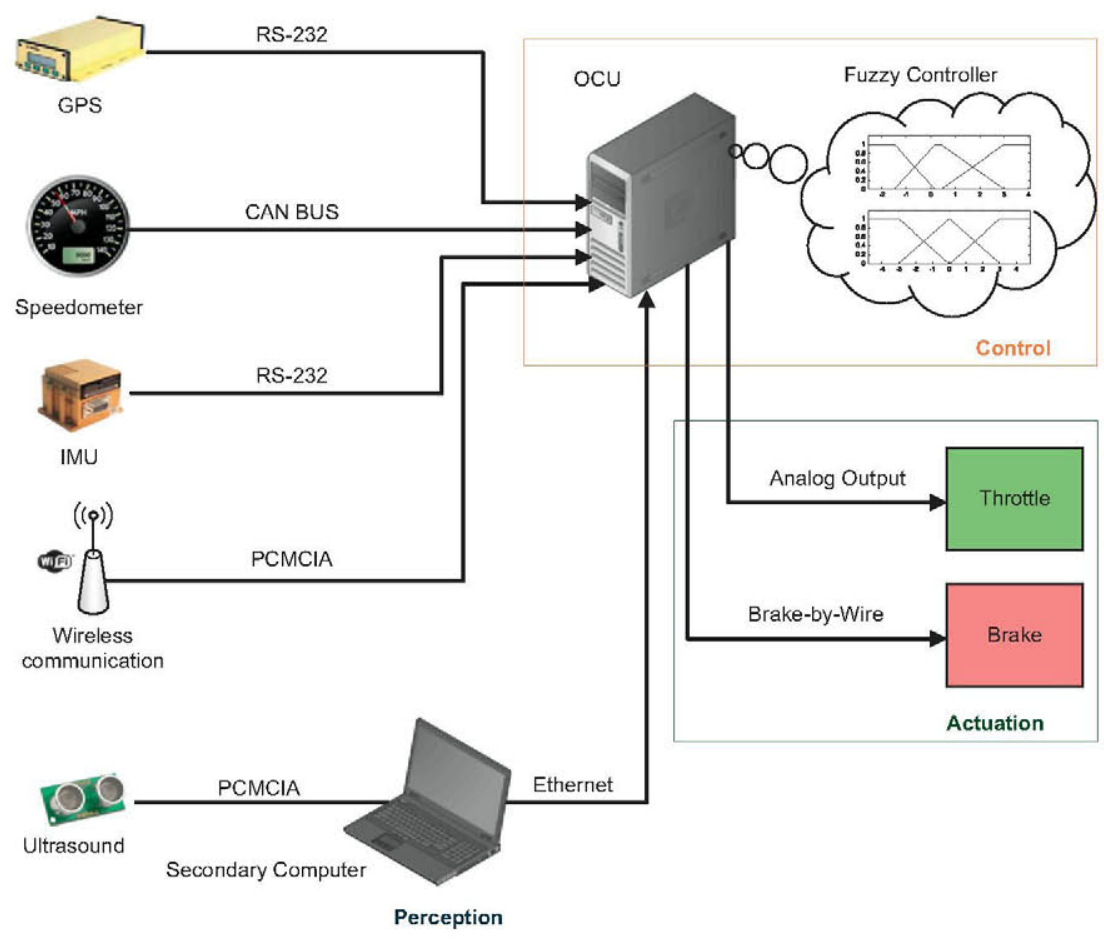

Fig. 1. On-board system for driving aid applications.

The third and last stage is electro-mechanical, responsible for converting the output generated by the fuzzy controllers into voltages that can then be sent to the actuators - the throttle and brake pedals. The actuator automation will be described in Section 6.

In the actual implementation, an on-board control unit (OCU) is located in the car's boot. It is in charge of collecting the information from the sensor systems. Specifically, an RS-232 connection is used for both the DGPS and the IMU positioning systems. A peripheral component interconnect (PCI) card is used to connect the PCMCIA Proxim Wireless ComboCard so as to acquire the information coming from other cars. With respect to the CAN bus, another PCMCIA CAN card is installed in the OCU. Finally, the information coming from the ultrasound sensor via the PCMCIA is received on a laptop computer which is responsible for processing the data received and sending the calculated distance to the OCU via an Ethernet connection. In this way, the integration of additional sensorial systems - cameras or lidars - can be easily carried out using Ethernet connections to send the relevant information to the OCU. Fig. 1 is a schematic diagram of the complete system.

\section{In-car sensors for leading vehicle detection}

Vehicular ad hoc networking will play a key role for future safety applications in road vehicles [19]. In this connection, we propose a V2V communication improvement to apply to ACC systems in order to provide a backup substitute when radar failures occur, together with a specific system to permit throttle and brake control when a preceding car is detected.

Using V2V, a vehicle can detect the position and movement of other vehicles up to $200 \mathrm{~m}$ away. Via accurate DGPS/IMU fusion and the data received through the CAN bus, the vehicle's position and speed can be communicated to other cars in the surrounding area so that they will also know its location, even when it is in a blind spot, halted ahead on the road but hidden from view, around a blind corner, or blocked by other vehicles. The vehicles can anticipate and react to changing driving situations and then warn the driver through visual or sound signals when situations of danger are detected. The system we are describing goes one step further and acts on the vehicle, controlling throttle and brake, even stopping it if necessary.

Systems based on visible light or radar technology can be replaced by a communication system with a better and significantly less costly way of sensing other vehicles around the car while it is being driven. In the present study, we used V2V communication data to provide the information needed to perform an autonomous ACC with NMV detection. To this end, a frame comprising three fields has been defined to exchange data among vehicles-leading and trailing. Two of the fields are the Universal Transverse Mercator (UTM) coordinates - North and East - in metres, and the other is the speed in kilometres per hour. This information is sent each control cycle. The control stage assesses these values in each control cycle, and, in the case that a traffic situation involving an NMV is detected, generates the output to the actuators in order to keep both the vehicle's occupants and the NMV safe. 
Table 1

Probability of failures in the communication system.

\begin{tabular}{lllll}
\hline No. of vehicles & 20 & 40 & 60 & 80 \\
\hline Prob. of failure & $3.29 \times 10^{-14}$ & $2.35 \times 10^{-7}$ & $4.04 \times 10^{-5}$ & $5.16 \times 10^{-4}$ \\
\hline
\end{tabular}
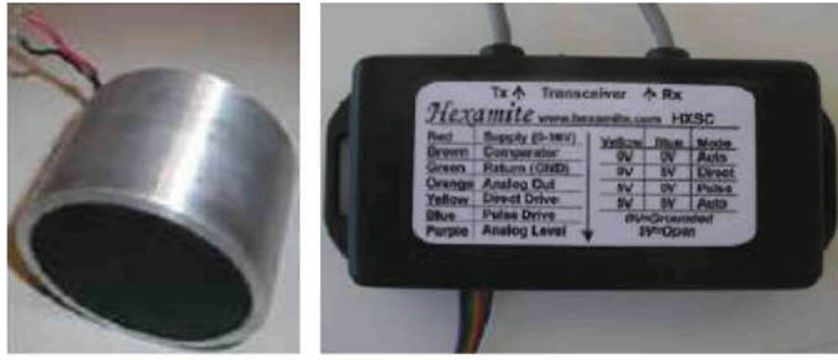

Fig. 2. Left: Ultrasound sensor. Right: Signal conditioner.

The V2V communication system has been extensively tested in many trials in the CAR facilities. During these trials, the unique communication failure detected was a loss of signal during one control cycle. This was reflected in two identical consecutive values of the variables. Our control system is able to cope with such a situation by deactivating the automatic systems that depend on communication if two or more consecutive failures are detected. A detailed theoretical study of the wireless communication system's behaviour and the probability of failures are found in [20]. A summary of the probability of failures in function of the number of vehicles can be found in Table 1 . One can appreciate how the probability of failure is low even for 100 vehicles in the surrounding area.

\section{In-car sensors for NMV detection}

Ultrasound sensors are currently used in parking aid systems for detection ranges of about $1 \mathrm{~m}$. The present proposed application uses them to detect NMVs in the road at a range of some $10 \mathrm{~m}$. This enables actuating the braking system to avoid a collision, or at least to reduce its severity. At these ranges, the factor that most affects ultrasound propagation is the turbulence caused by wind and vehicle movement.

For this application, we chose a $43 \mathrm{kHz}$ long-range, narrow-beam, Hexamite ultrasound sensor, acting as transmitterreceiver. The beam width is $8.5^{\circ}$ at $-3 \mathrm{~dB}$. It is shown together with its signal conditioner in Fig. 2 . The conditioner's mission is to excite the sensor with a pulse train, and then filter and amplify the received signal.

Fig. 3 illustrates the sensitivity of the sensor when excited with a train of 50 pulses of 20 volts peak-to-peak, with a flat circular object of $26 \mathrm{~cm}$ radius placed at a distance of $90 \mathrm{~cm}$, together with the beam width.

Fig. 4 shows the sensitivity, impedance, and phase angle as functions of frequency. The characteristic values at $43 \mathrm{kHz}$ are about $-54 \mathrm{~dB}, 700 \mathrm{ohm}$, and $-20^{\circ}$, respectively.

For the proposed application, the ultrasound sensor is connected to a computer through its signal conditioner and a data acquisition card. Through this latter, the computer applies a train of 60 pulses at a frequency of $43 \mathrm{kHz}$ to the ultrasound sensor, and then the received signal is digitized by the acquisition card and stored in memory for further processing. This processing is performed by software and consists of the following steps:

1. Reducing noise via a band-pass second-order Butterworth filter with lower and upper cut-off frequencies of 42 and $44 \mathrm{kHz}$, respectively. The filtered signal $y$ is obtained from the sampled ultrasound signal $x$ according to the following expression:

$$
\begin{aligned}
y_{n}= & 0.00104438 x_{n}-0.00208876 x_{n-2}+0.00104438 x_{n-4}+0.57951312 y_{n-1} \\
& -1.99053196 y_{n-2}+0.55302075 y_{n-3}-0.91070675 y_{n-4}
\end{aligned}
$$

2. Obtaining the envelope of the filtered signal $y$ via the Hilbert transform, as is standard in signal processing. The Hilbert transform $y$ of an amplitude modulated sinusoidal signal $y$ is an identical signal but $90^{\circ}$ out of phase. Using the two, one creates an analytic signal $y_{a}(t)=y(t)+i^{\prime} y=a(t)^{i \phi(t)}$ whose magnitude $a(t)$ is the envelope of the original signal.

3. Getting the third power $a_{a}(t)=a^{3}(t)$ of the envelope obtained in the previous step. This selectively amplifies those parts of the signal with greatest amplitude, thus improving the discrimination between the signal and the noise still present, 


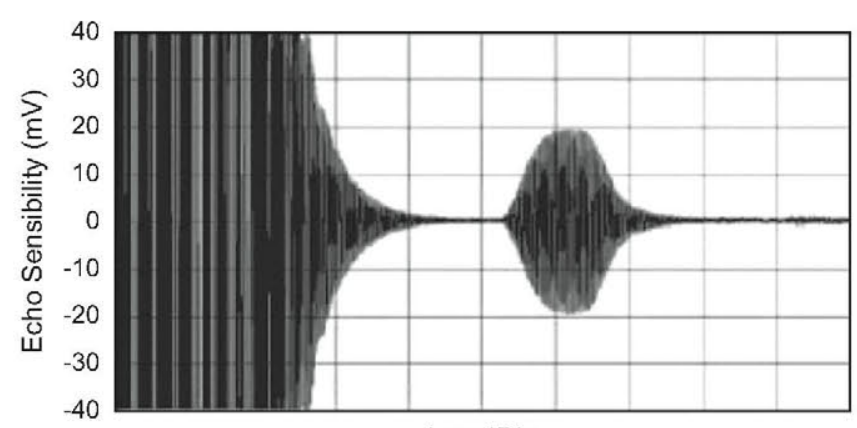

$1 \mathrm{~ms} /$ Div.

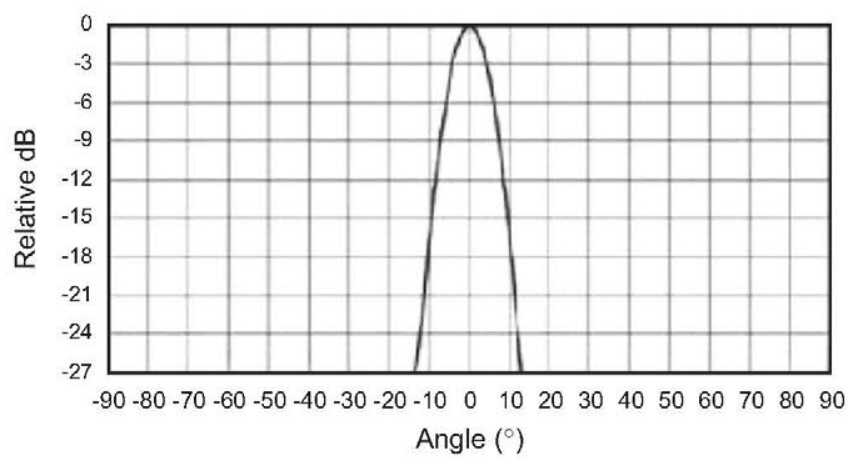

Fig. 3. Top: Sensitivity of the sensor excited with a train of 50 pulses of 20 volts peak-to-peak, with a flat circular object of $26 \mathrm{~cm}$ radius placed at a distance of $90 \mathrm{~cm}$. Bottom: Beam width.
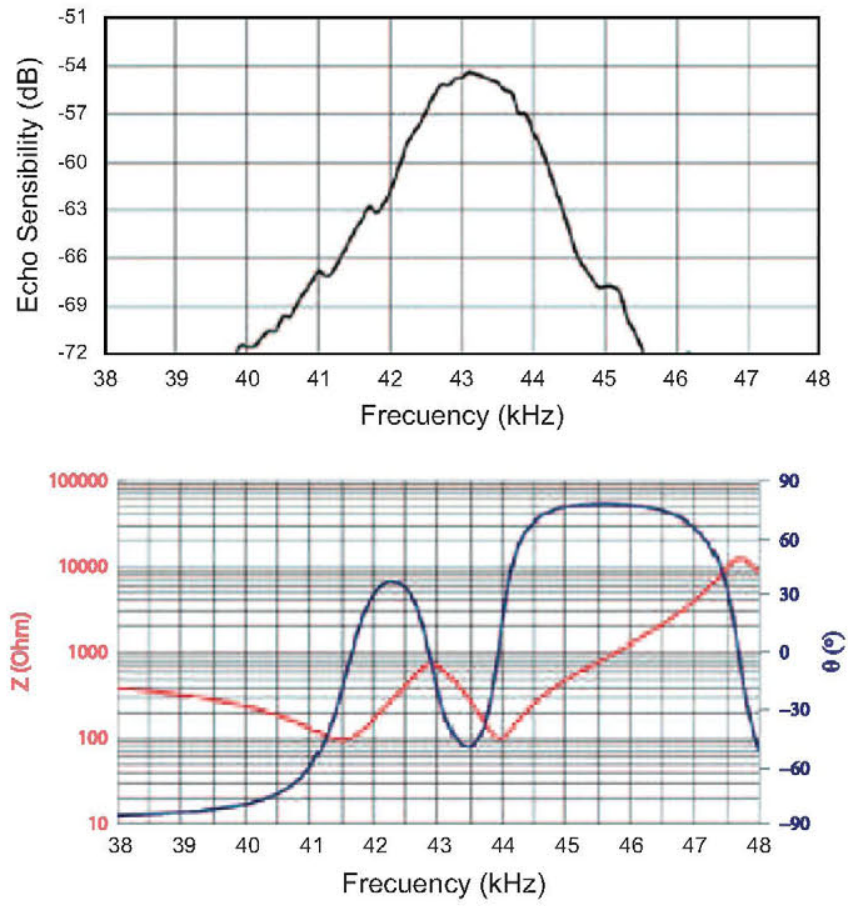

Fig. 4. Top: Sensitivity as a function of frequency. Bottom: Impedance and phase as functions of frequency.

due, for example, to disturbances caused by air turbulence and vibrations of the vehicle, and even to the echoes produced by irregularities in the roadway.

4. The final signal $s(t)$ is obtained by multiplying $a_{a}(t)$ by an exponentially increasing gain $g(d)$ to compensate for the attenuation of sound during propagation through the air-i.e., $s(t)=a_{a}(t) g(t)$. Here, $d$ is the distance travelled by the 
ultrasound pulse from the transmitter to the obstacles in the path, and is given by the expression $d=c_{s} t / 2$, where $t$ is the time-of-flight and $c_{s}$ is the speed of sound. The mathematical expression used for this gain is $g(d)=\exp (0.8 d)$ or equivalently $g(t)=\exp \left(0.4 c_{s} t\right)$.

After each step, the signal is normalized by dividing each sample by the maximum value of the signal.

Signal acquisition and processing can be performed with a conventional computer at a rate of about $10 \mathrm{~Hz}$, so that the system is able to react much more quickly than a human driver would. Fig. 5 shows the result of the different processing steps.

In the figure, echoes can be seen that are produced by a vehicle located about $7.5 \mathrm{~m}$ in front of the sensor, and a pedestrian at about $4.75 \mathrm{~m}$. To determine the time of arrival of the echoes, the signal is compared with a reference level of $2 \times 10^{-4}$, experimentally adjusted to minimize the number of detection errors. The elapsed time between the sensor trigger and the arrival of an echo is known as the "time of flight". One observes that, despite being farther away, the vehicle produces a larger echo than an NMV due to its greater reflecting surface and reflectivity.

The distance to the nearest object is obtained by multiplying the time of flight of the first detected echo by the speed of sound in air (about $344 \mathrm{~m} / \mathrm{s}$ at $20^{\circ} \mathrm{C}$ ), and dividing by two to take into account that the signal has travelled from sensor to object and back.

The main problems encountered are false detections when there are no obstacles on the road, which would activate the brake unnecessarily, and non-detection of obstacles that really exist, which could allow a collision. These detection errors are mainly caused by turbulence due to wind and vehicle movement, vibration, and irregularities in the road, and are more frequent the greater the detection distance. If these problems occur in only a small fraction of detections, their effect on
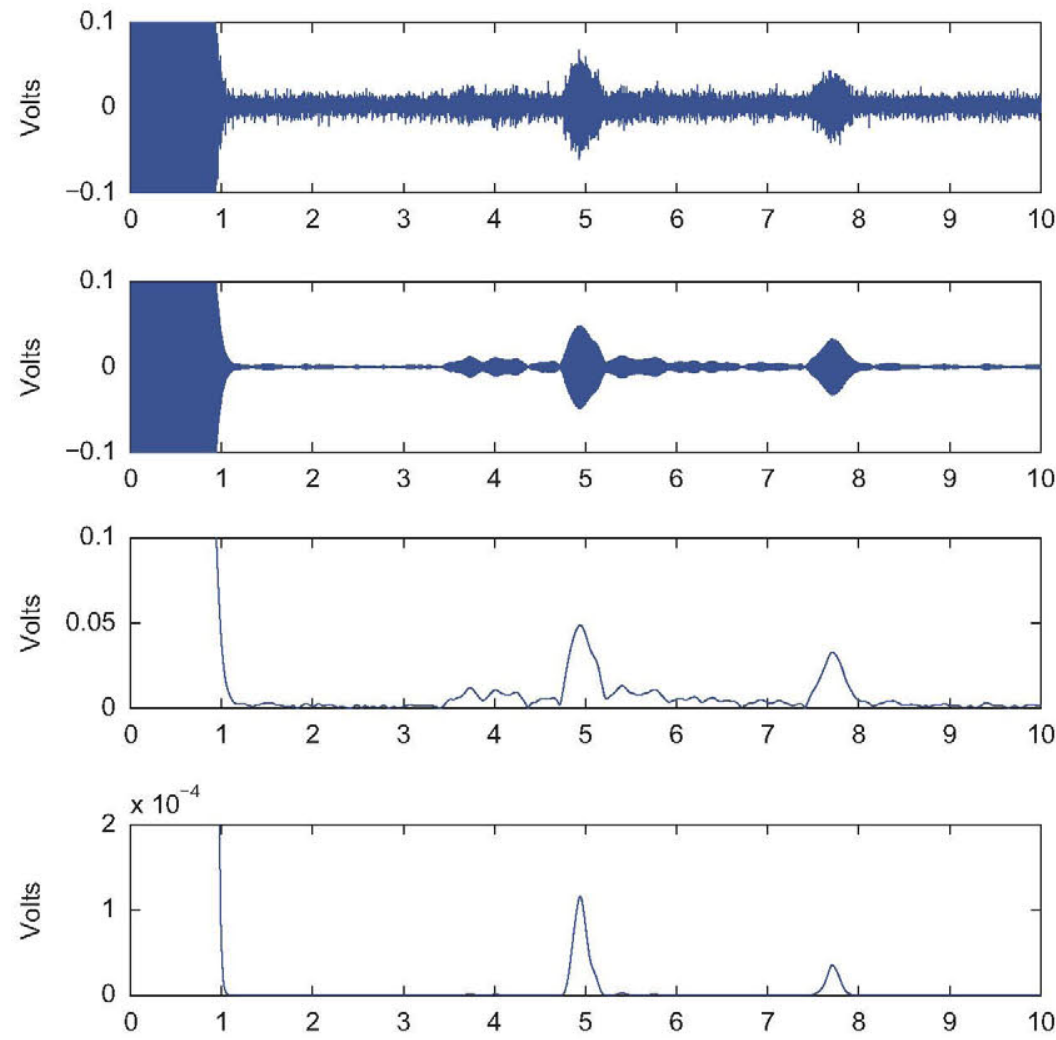

(4)

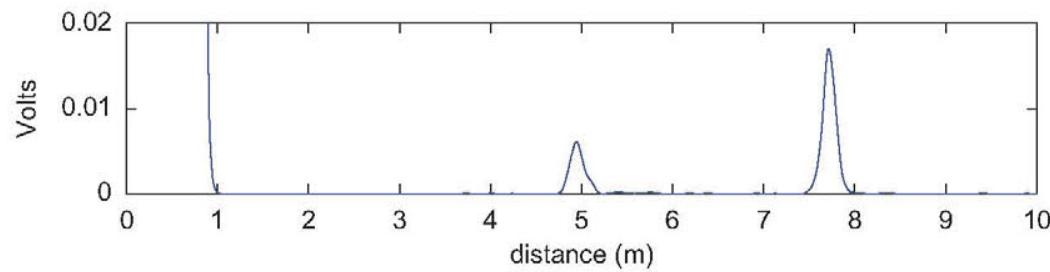

(5)

Fig. 5. From top to bottom: (1) ultrasound signal $x(t)$ received by the sensor; (2) filtered signal $y(t)$ obtained in step $1 ;(3)$ envelope $a(t)$ obtained in step 2 ; (4) envelope raised to the third power $a_{a}(t)$ obtained in step 3; (5) amplified signal $s(t)$ obtained in step 4. 
braking is almost imperceptible for vehicle's passengers because of their short duration, i.e., the vehicle dynamics is not modified for isolated failures in detection. Only when there is a major fraction of such failures or a number of consecutive failures occur will their effect have the potential to harm the system's performance.

To check the reliability of the system, several experiments were performed, limiting the measuring distance to $11 \mathrm{~m}$ in the sense that a measured distance of $11 \mathrm{~m}$ means that no object has been detected.

In the first experiment, 100 measurements were made with the sensor motionless and no obstacles in front. Fig. 6 shows the results. As can be seen, only one false echo was detected.

In the second experiment, the sensor was placed on a vehicle moving towards and away from another parked vehicle, and the distance was again measured 100 times. Fig. 7 shows the results. One can appreciate one failure during the experiment. Ultrasound errors in outdoor environments have been studied in previous work [21,22]. In brief, two main drawbacks are responsible for ultrasound failures:

1. A velocity gradient is established that causes the refraction of acoustic signals that propagate upwind or downwind. For propagation distances of a few metres - as in our case - this effect is not very important, and only causes a small additional attenuation of the received signal.

2. Presence of turbulence: vortices associated with turbulences act as scattering centres of acoustic energy, and their randomness makes this phenomenon particularly difficult to compensate.

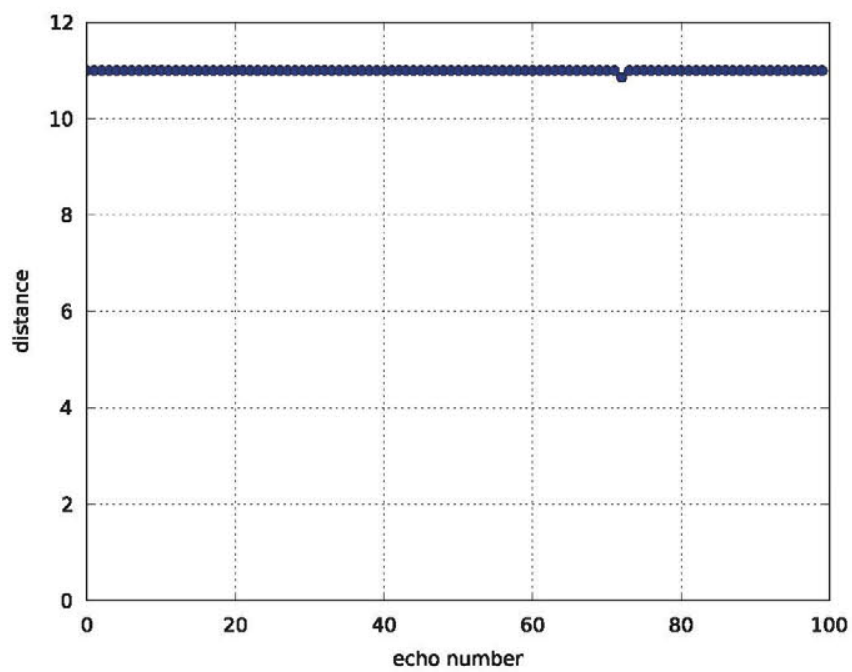

Fig. 6. Distances measured with no object in front.

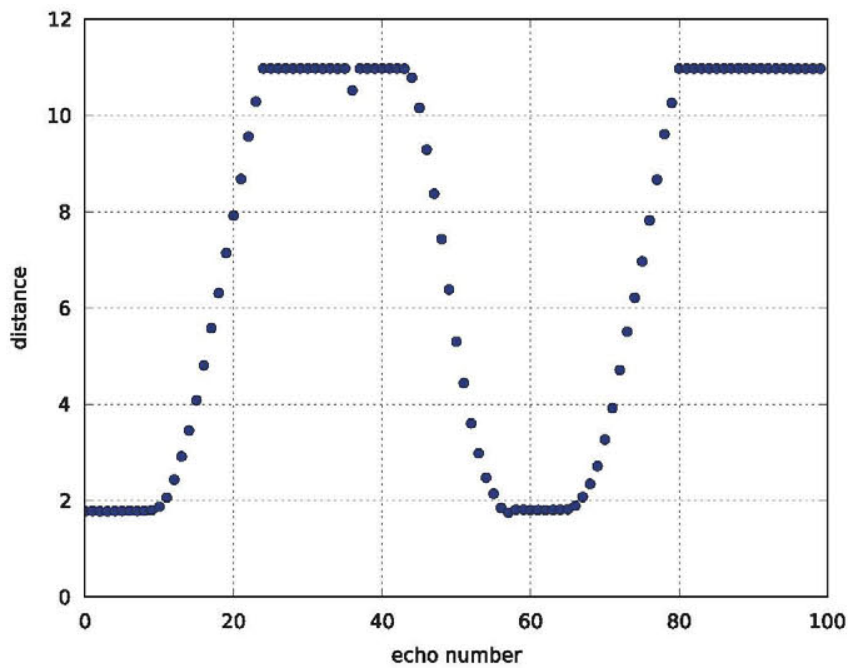

Fig. 7. Distance measured when moving towards and away from another parked vehicle. 
To analyze the influence of wind speed on the ultrasonic system, a third experiment has been conducted in which a pedestrian has been placed at different distances from the transmitter - 2.5, 5.0, 7.5 and $10 \mathrm{~m}$ - with wind speeds from 5 to $35 \mathrm{~km} / \mathrm{h}$ in $5 \mathrm{~km} / \mathrm{h}$ intervals. Taking into account DGPS errors - $50 \mathrm{~cm}$ per car, measurements with an error lower than $10 \%$ are considered correct - same range of tolerance than DGPS. Experimental results are shown in Table 2 . One can appreciate how the obtained results are good enough for the goal of detecting pedestrians and measuring the distance at which they are.

Remark that although the trailing vehicle motion may generate turbulences at the rear, its effect on the ultrasonic wave is insignificant with respect to wind gusts considered in the experiment.

The fourth experiment consisted of 100 measurements of the distance between the sensor and an NMV - in this case a pedestrian - in front, both motionless, and repeating the experiment for various distances between 2 and $10 \mathrm{~m}$. The same experiment was subsequently performed with a vehicle replacing the pedestrian. Fig. 8 shows the results. One observes how the scatter of the data increases with distance due to the increasing influence of the wind.

For each set of 100 measurements, the median value (taken to be the actual measured distance), the standard deviation, and the number of measurements that differed from the median by more than $10 \%$ (considered as incorrect measures) were calculated. Moreover, the mean and maximum relative difference of the whole set of measures with respect to the reference value are also computed to have an indicator of the measurement variability. The results are given in Table 3 . One observes that, in detecting the vehicle, there were zero errors for all distances, i.e., 100\% correct detection was attained (although the standard deviation increased with distance). For the case of the pedestrian, however, the number of detection failures increased steadily with distance, reaching a $13 \%$ failure rate at $10 \mathrm{~m}$. The failure rate considering all the measurements was 6.8\% (61 failures in 900 measurements). There were no false detections with the fixed reference level applied - these would be expected if the reference level were reduced or the intensity of the turbulences increased.

One can conclude that the system is useful for the detection of pedestrians and other obstacles on the road, since the probability of two consecutive detection failures is very low, and because the short time interval between measurements means that the effect of any failure is filtered out by the dynamics of the vehicle.

Note that the system is not designed to distinguish between different kinds of NMV - pedestrian, cyclist, and so on but to distinguish between vehicles and NMV thanks to the comparison with the distance measured by the wireless communication system. Pedestrians were selected as the example type of NMV because they are the hardest to detect.

Table 2

Percentage of correct distance measurements as function of distance and wind speed.

\begin{tabular}{lllll}
\hline Wind speed $(\mathbf{k m} / \mathbf{h})$ & \multicolumn{4}{l}{ Distance $(\mathbf{m})$} \\
\cline { 2 - 5 } & 2.5 & 5.0 & 7.5 & 10.0 \\
\hline $5-10$ & 100 & 100 & 100 & 92.3 \\
$10-15$ & 100 & 100 & 100 & 86 \\
$15-20$ & 100 & 100 & 99.4 & 83 \\
$20-25$ & 100 & 100 & 99.5 & 83.8 \\
$25-30$ & 100 & 100 & 98.5 & 67.7 \\
$30-35$ & 100 & 100 & 100 & 65 \\
\hline
\end{tabular}
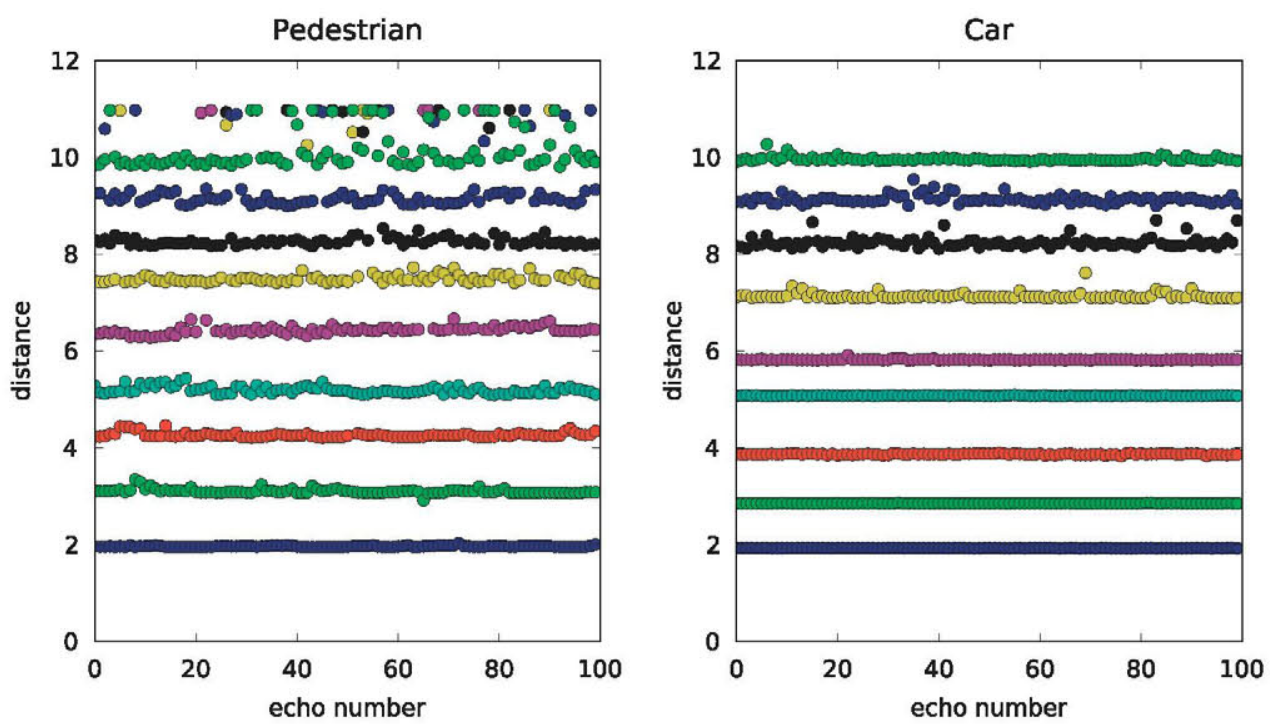

Fig. 8. Left: Distances measured to a pedestrian. Right: Distances measured to a vehicle. 
Table 3

Summary statistics of the measurements made for a pedestrian and a vehicle.

\begin{tabular}{|c|c|c|c|c|c|c|c|c|c|}
\hline \multirow{2}{*}{$\begin{array}{l}\text { Measurements } \\
\text { Median }\end{array}$} & \multicolumn{9}{|c|}{ Pedestrian } \\
\hline & 1.962 & 3.090 & 4.254 & 5.174 & 6.428 & 7.468 & 8.254 & 9.145 & 9.959 \\
\hline S.D. & 0.013 & 0.050 & 0.069 & 0.074 & 0.991 & 0.953 & 0.862 & 0.577 & 0.400 \\
\hline No. errors & 0 & 0 & 1 & 0 & 5 & 9 & 12 & 13 & 13 \\
\hline Max. rel. diff. & 3.3 & 8.2 & 157.9 & 4.9 & 70.7 & 46.9 & 32.9 & 20.0 & 10.2 \\
\hline \multirow[t]{2}{*}{ Mean. rel diff. } & 0.5 & 0.9 & 2.3 & 1.1 & 4.3 & 4.6 & 4.4 & 3.2 & 2.7 \\
\hline & \multicolumn{9}{|l|}{ Car } \\
\hline Median & 1.928 & 2.851 & 3.867 & 5.075 & 5.819 & 7.114 & 8.215 & 9.113 & 9.949 \\
\hline S.D. & 0.002 & 0.002 & 0.011 & 0.005 & 0.011 & 0.068 & 0.112 & 0.085 & 0.047 \\
\hline No. errors & 0 & 0 & 0 & 0 & 0 & 0 & 0 & 0 & 0 \\
\hline Max. rel. diff. & 0.3 & 0.2 & 1.1 & 0.4 & 1.6 & 7.0 & 5.9 & 4.7 & 3.2 \\
\hline Mean rel. diff. & 0.08 & 0.06 & 0.22 & 0.08 & 0.09 & 0.44 & 0.82 & 0.63 & 0.24 \\
\hline
\end{tabular}

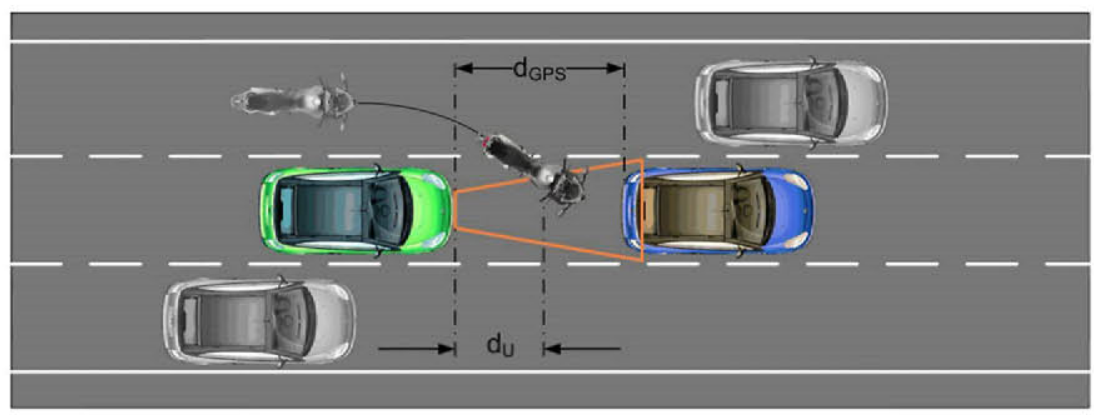

Fig. 9. Schematic diagram of a risky traffic situation.

\section{Control system}

In this section, we shall describe the control algorithm used in the operation of the ACC + NMV detection system. In spite of the highly nonlinear behaviour of a vehicle, a human learns to drive it on the basis of experience. We use this intuitive human experience as expert knowledge in order to design a fuzzy controller capable of carrying out the vehicle's speed control taking into account both the leading vehicle and any intervening NMV.

Fig. 9 represents a typical risk situation in urban traffic. We here consider three congested lanes with vehicles driving in the same direction, as occurs in large cities. In this traffic situation, an NMV overtakes other vehicles, with frequent lane changes, thus increasing the risk of collision. Given these premises, our control has to satisfy the following requirements:

- Maintain the distance with the preceding car. This is done with positioning data of the preceding car obtained using the wireless communications systems $\left(d_{G P S}\right)$.

- Identify any object that may appear between the two cars, and act to avoid a collision. This is done with data obtained from the ultrasound sensor $\left(d_{U}\right)$.

Three variables are considered to perform the control. The first is the inter-vehicle distance error, which is the difference between the target reference distance, set at $7 \mathrm{~m}$, and the actual distance obtained via wireless communication $\left(d_{G P S}\right)$. The second is the difference in distance (ultrasound $\left.d_{e r r o r}\right)$ between the values obtained via wireless communications and via ultrasound so as to determine when any NMV is detected, and is defined as

$$
\text { ultrasound }_{\text {error }}=d_{G P S}-d_{U}
$$

Finally, the vehicle's speed is taken into account in order to decide whether to increase or decrease actions on the pedals. The greater the speed, the greater the control action.

The fuzzy controller developed will be responsible for managing the brake and throttle pedals - i.e., the longitudinal control - in order to modify the autonomous vehicle's speed. AUTOPIA program has previously developed an experimental fuzzy system [23], which is an inference motor with a straight-forward natural-language-based input language. It works with Mamdani's inference method, with singleton-type membership functions to codify the output variables.

Fig. 10 shows the membership function definitions for each of these three input variables. For the variable Velocity, there are three membership function definitions - Low, Medium, and High - thus assigning the vehicle's speed in traffic jams to one of three categories. Backward driving is not considered so values lower than $0 \mathrm{~km} / \mathrm{h}$ are not allowed. The Distance Error variable also has three membership functions. 'Negative' and 'Positive' membership functions to reflect in 

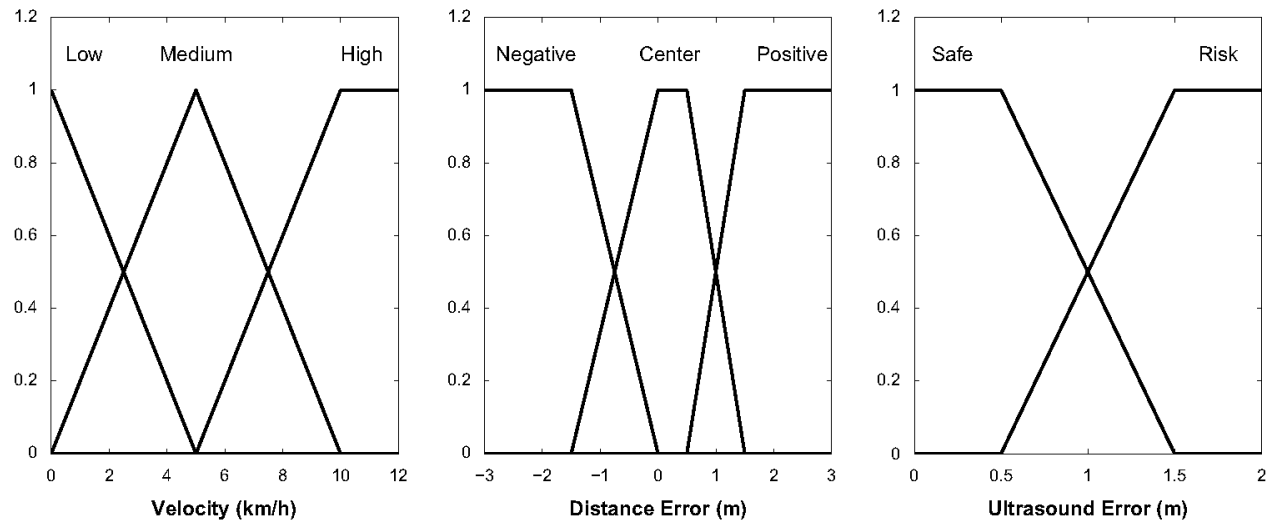

Fig. 10. Membership functions for the input variables.

Table 4

Rule base for ultrasound error equal to safe.

\begin{tabular}{llll}
\hline Velocity & \multicolumn{3}{l}{ Distance error } \\
\cline { 2 - 4 } & Negative & Centre & Positive \\
\hline Low & 0.3 & 0 & -0.15 \\
Medium & 0.15 & 0 & -0.15 \\
High & 0.15 & 0 & -0.15 \\
\hline
\end{tabular}

Table 5

Rule base for ultrasound error equal to risk.

\begin{tabular}{llll}
\hline Velocity & \multicolumn{2}{l}{ Distance error } \\
\cline { 2 - 4 } & Negative & Centre & Positive \\
\hline Low & -0.15 & -0.15 & -0.15 \\
Medium & -0.15 & -0.3 & -0.3 \\
High & -0.3 & -0.3 & -0.3 \\
\hline
\end{tabular}

general terms the need to increase or decrease the distance, respectively; and a central membership function with trapezoidal shape. The reason is to consider measurement errors on the part of DGPS and ultrasound. In this way, a softer action over vehicle's actuators is achieved while keeping safe. Finally, the Ultrasound Error variable, that represents the difference between the actual distance via wireless communication and via ultrasound sensor, has two membership functions reflecting the possible risk situation involving an NMV detection.

As output, a normalized action on the throttle and brake pedals is defined. This is done through the variable Pedal, defined between [ $-1,1]$, where -1 indicates maximum action applied to the brake and 1 indicates maximum action applied to the accelerator pedal. Tables 4 and 5 present the rule base generated.

In brief, the control system works as follows: When a vehicle equipped with the system described is on the road, only other vehicles and NMVs can be detected. Taking this into account, V2V communication is used to detect vehicles in the vicinity. If the value of the distance obtained via ultrasound is different from that obtained from the GPS, one may assume that an NMV has appeared between the two cars. The rule base generated takes these cases into account so as to act on the brake or the throttle according to the values given by the perception stage.

\section{Automation of the actuators}

A convertible Citroën C3 Pluriel (see Fig. 11) was used to test the driving aid system that has been presented above. In equipping the car with an automatic driving aid system, the actuators needed to be modified to permit their automatic control. This section describes the hardware modifications made to the brake and throttle pedals.

\subsection{Throttle}

The throttle is controlled using a PCMCIA analogue card. The Citroën control unit receives an analogue signal whose value is modified according to the pressure applied on the throttle pedal. An acquisition data card is used to generate the 
maximum and minimum voltage values to apply to the throttle pedal. A switch was installed in order to choose between the signal generated by the throttle pedal or by the PCMCIA analogue card, so that either the original system or the computer controlled system can act on the vehicle.

\subsection{Brake}

Since the brake pedal is critical, a brake-by-wire system capable of co-existing with it was developed. To this end, an electro-hydraulic braking system was installed in the vehicle. This system is connected to the hydraulic brake circuit of the car before the anti-lock brake system (ABS) using shuttle valves. The OCU is capable of controlling this actuator via a CAN device that generates two signals: an analogue signal that regulates the pressure value and a digital signal to logically activate it [24]. The reaction time on the part of the electro-hydraulic braking system is equal to $30 \mathrm{~ms}$ in the first control cycle - because a spool directional valve - and lower than $10 \mathrm{~ms}$ in the next control cycles - because a electro-proportional pilot. Maximum deceleration are higher than $10 \mathrm{~m} / \mathrm{s}^{2}$ but it was limited to increase passenger's comfort.

\section{In-circuit results}

Numerous experiments were performed to check the behaviour of the control system. To this end, CAR's private driving circuit was used to validate the system in a real traffic environment. Two vehicles (see Fig. 11), one of them the convertible Citroën C3 Pluriel and the other a Citroën C3, were used in this phase.

Fig. 12 illustrates the behaviour of the ACC +NMV-detection fuzzy controller emulating a traffic jam in an urban environment-speeds less than $15 \mathrm{~km} / \mathrm{h}$. The top plot shows the speeds of the two cars, the grey curve representing the leading vehicle, and the black curve the trailing one. The next to top plot depicts the trailing car's acceleration - solid grey line - and jerk - solid black line - to evaluate the comfort of the control. The next to bottom plot shows the distance separating the two cars as measured using the DPGS (black curve) and the ultrasound sensor measurements (grey curve). The bottom plot depicts the normalized fuzzy output that will act on the brake and throttle pedals.

At the beginning, both vehicles are motionless, with a separation equal to $4 \mathrm{~m}$. During these first $5 \mathrm{~s}$, one can appreciate small errors - less than $0.5 \mathrm{~m}$ - in the ultrasound distance measurements with respect to the DGPS measured distance. Then the leading vehicle starts and the trailing one attempts to follow it. Given the low speeds, minimal actions on the throttle or brake - less than $30 \%$ - cause considerable changes in the distance between vehicles. The gap fluctuates around $7 \mathrm{~m}$. At about $25 \mathrm{~s}$, a significant change in the inter-distance obtained via the ultrasound sensor is registered. The control system detects an NMV between the two vehicles, and stops the autonomous car. The trailing vehicle's speed becomes zero when the distance to the NMV is slightly more than $2 \mathrm{~m}$.

Once the NMV collision has been avoided, the trailing vehicle continues on its way. One observes in the figure that, at this moment, the distance obtained via the ultrasound sensor is equal to $10 \mathrm{~m}$ - the maximum range of the ultrasound sensor. While ultrasound measurements are not available, it means that NMV has not been detected and the distance with the leading vehicle is out of range for the ultrasound sensor. In these cases, the value of the distance measures by the ultrasound is set to the distance measures by the DGPS. So the value of the Ultrasound Error variable is zero until either gap distance is lower than $10 \mathrm{~m}$ or a NMV appears in the vehicle's trajectory. When the separation between the two vehicles is again less than $10 \mathrm{~m}$, the ultrasound sensor measurements are computed anew. At around $45 \mathrm{~s}$, another NMV is detected, again stopping the car.

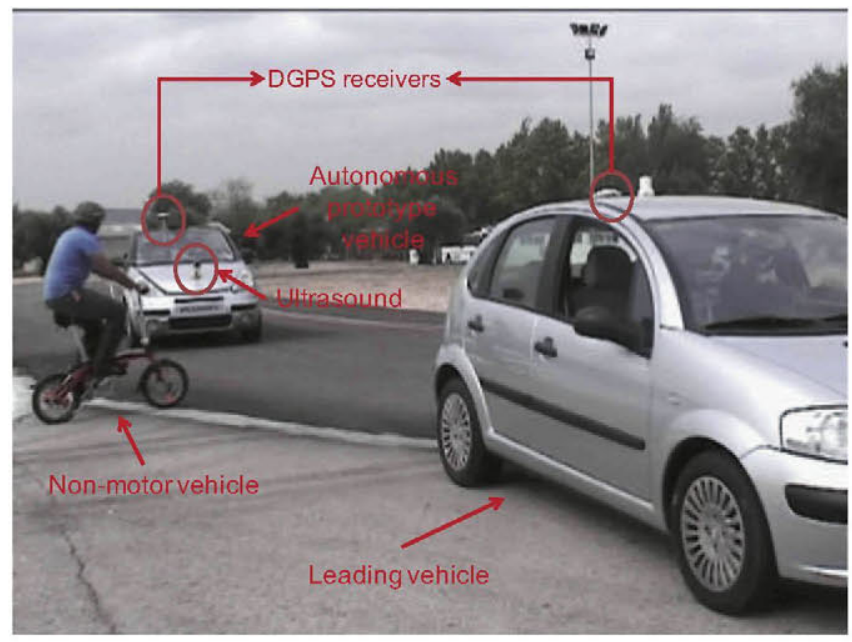

Fig. 11. Autonomous prototype vehicle. 

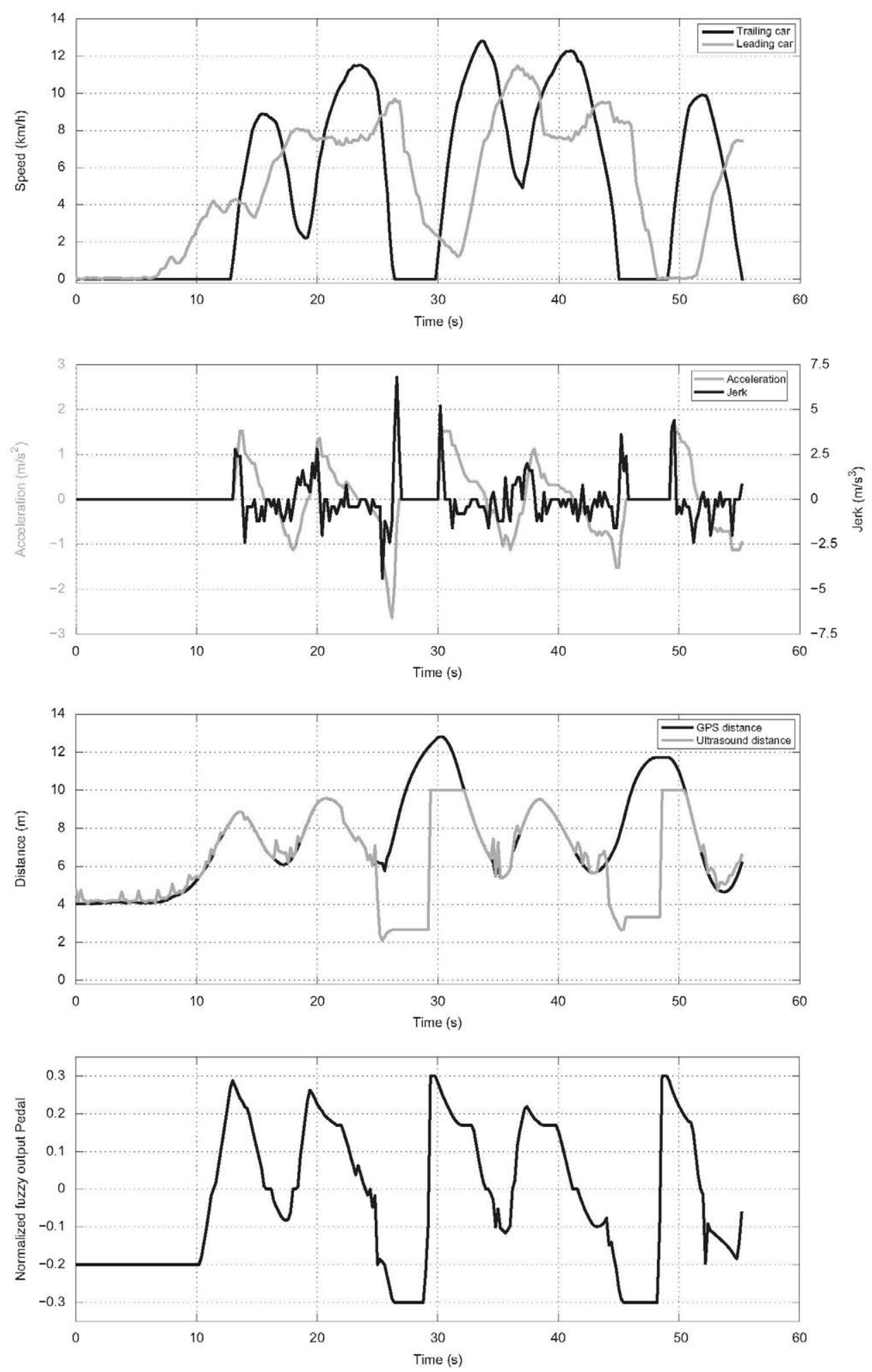

Fig. 12. Experimental results.

With respect to the comfort of the trailing vehicle's occupants, one observes that the acceleration is less than $\left|2 \mathrm{~m} / \mathrm{s}^{2}\right|-$ the maximum acceleration for comfort is typically fixed at this value [25] - throughout the experiment. A deceleration slightly greater than $2 \mathrm{~m} / \mathrm{s}^{2}$ in value occurs when the first NMV is detected for extra safety. This behaviour is reflected in 
the jerk measurements. More experiments of this type were conducted, also illustrating the good behaviour of the designed controller.

\section{Conclusions and next steps}

Traffic accidents in urban environments constitute a major problem for governments. This paper has presented a solution for traffic jam situations in which NMVs are walking or being driven in and out of the lines of motor vehicles. The system consists of combining DGPS with wireless communication to control the gaps between vehicles, and using an ultrasound sensor for NMV detection. Experiments with real cars gave encouraging results. They demonstrated that environment perception using sensor systems and a controller capable of managing the vehicle's actuators can considerably reduce urban accidents, whether vehicle-vehicle or vehicle-NMV.

The results are therefore promising, but there are still several issues to be investigated. In particular, neither curved stretches of road have been considered for the NMV detection, nor tunnels for vehicle-vehicle following.

\section{Appendix A. Supplementary material}

Supplementary data associated with this article can be found in the online version of http://dx.doi.org/10.1016/j.ymssp. 2012.04.008.

\section{References}

[1] G. Reya, D. Claira, M. Foglia, F. Bernardin, Reliability analysis of roadway departure risk using stochastic processes, Mech. Syst. Signal Process. 25 (2011) 1377-1392.

[2] B. Asadi, A. Vahidi, Predictive cruise control: utilizing upcoming traffic signal information for improving fuel economy and reducing trip time, IEEE Trans. Control Syst. Technol. (2010) 1-9. (early access).

[3] M. Bansal, A. Das, G. Kreutzer, J. Eledath, R. Kumar, H. Sawhney, Vision-based perception for autonomous urban navigation, in: Proceedings of the 11th International IEEE Conference on Intelligent Transportation Systems ITSC 2008, 2008, pp. 434-440.

[4] E. Onieva, V. Milanés, C. González, T. de Pedro, J. Perez, J. Alonso, Throttle and brake pedals automation for populated areas, Robotica 28 (2010) 509-516.

[5] R. Schöneburg, T. Breitling, Enhancement of active and passive safety by future pre-safe systems, in: 19th ESV Conference, 2002.

[6] ERSO, Traffic Safety Basic Facts 2007, Technical Report, European Transport Commission, 2007.

[7] M. Peden, R. Scurfield, D. Sleet, D. Mohan, A. Hyder, E. Jarawan, C. Mathers, World Report on Road Traffic Injury Prevention, Technical Report, World Health Organization, 2004

[8] M. Distner, M. Bengtsson, T. Brobert, L. Jakobsson, City safety-a system addressing rear-end collisions at low speeds, in: 21 st Enhanced Safety Vehicles Conference, 2009.

[9] E. Coelingh, A. Eidehall, M. Bengtsson, Collision warning with full auto brake and pedestrian detection-a practical example of automatic emergency braking, in: Proceedings of the 13th International Intelligent Transportation Systems (ITSC) IEEE Conference, 2010, pp. 155-160.

[10] W. Wijesoma, K. Kodagoda, A. Balasuriya, Road-boundary detection and tracking using ladar sensing, IEEE Trans. Robotics Autom. 20 (2004) 456-464.

[11] S. Park, T. Kim, S. Kang, K. Koo, A novel signal processing technique for vehicle detection radar, in: IEEE MTT-S International Microwave Symposium Digest, vol. 1, Institute of Electrical and Electronics Engineers, 2003, pp. 607-610.

[12] S. Zehang, G. Bebis, R. Miller, On-road vehicle detection: a review, IEEE Trans. Pattern Anal. Mach. Intell. 28 (2006) 694-711.

[13] M. Bertozzi, A. Broggi, C. Caraffi, M.D. Rose, M. Felisa, G. Vezzoni, Pedestrian detection by means of far-infrared stereo vision, Comput. Vis. Image Understand. 106 (2007) 194-204.

[14] D. McNamara, B. Tucker, R. Kwiecinski, Automotive obstacle detection systems: a survey of design requirements and vehicle integration issues, SAE International, 1998.

[15] V. Milanés, J.E. Naranjo, C. Gonzánez, J. Alonso, T. de Pedro, Autonomous vehicle based in cooperative GPS and inertial systems, Robotica 26 (2008) 627-633.

[16] V. Milanés, E. Onieva, J. Pérez, T. de Pedro, C. González, Control de velocidad adaptativo para entornos urbanos congestionados, Rev. Iberoam. Autom. Inf. Ind. 6 (2009) 66-73.

[17] V. Milanés, J. Pérez, E. Onieva, C. González, Controller for urban intersections based on wireless communications and fuzzy logic, IEEE Trans. Intell. Transp. Syst. 11 (2010) 243-248.

[18] V. Milanés, E. Onieva, B. Vinagre, C. González, J. Pêrez, J. Alonso, Sistema de asistencia a la conducción basado en una red de comunicaciones de bajo coste, DYNA 85 (2010) 245-254.

[19] H. Chenga, H. Shana, W. Zhuan, Infotainment and road safety service support in vehicular networking: from a communication perspective, Mech. Syst. Signal Process. (2011).

[20] V. Milanês, E. Onieva, J. Pérez, J. Simó, C. Gonzâlez, T. de Pedro, Making transport safer: a V2V-based automated emergency braking system, Transport 26 (2011) 290-302.

[21] F. Âlvarez, J. Ureña, A. Hernández, M.M.J. García, Influence of atmospheric refraction on the performance of an outdoor ultrasonic pulse compression system, Appl. Acoust. 69 (2008) 994-1002.

[22] F. Álvarez, J. Ureña, M. Mazo, A. Hernández, J. García, C. de Marziani, High reliability outdoor sonar prototype based on efficient signal coding, IEEE Trans. Ultrason. Ferroelectr. Freq. Control 53 (2006) 1862-1872.

[23] R. Garcia, T. de Pedro, First application of the Orbex coprocessor: control of unmanned vehicles, Mathware Soft Comput. 2/3 (2000) 265-273.

[24] V. Milanés, C. González, J. Naranjo, E. Onieva, T. De Pedro, Electro-hydraulic braking system for autonomous vehicles, Int. J. Autom. Technol. 1 (2010) $89-95$.

[25] BECHTEL, Compendium of Executive Summaries from the Maglev System Concept Definition Final Reports, Technical Report, U.S. Department of Transportation, 1993. 